\title{
Asymptotic theory of wall-attached convection in a horizontal fluid layer with a vertical magnetic field
}

F. H. Busse

Citation: Physics of Fluids 20, 024102 (2008); doi: 10.1063/1.2837175

View online: https://doi.org/10.1063/1.2837175

View Table of Contents: http://aip.scitation.org/toc/phf/20/2

Published by the American Institute of Physics

\section{Articles you may be interested in}

Onset of plane layer magnetoconvection at low Ekman number

Physics of Fluids 27, 106602 (2015); 10.1063/1.4934532

Rayleigh-Bénard convection in liquid metal layers under the influence of a vertical magnetic field

Physics of Fluids 13, 3247 (2001); 10.1063/1.1404385

Onset of Rayleigh-Bénard convection for intermediate aspect ratio cylindrical containers

Physics of Fluids 29, 024107 (2017); 10.1063/1.4976543

Stability of convection rolls in the presence of a vertical magnetic field

The Physics of Fluids 25, 931 (1982); 10.1063/1.863845

Experimental and numerical study of Rayleigh-Bénard convection affected by a rotating magnetic field Physics of Fluids 11, 853 (1999); 10.1063/1.869957

Low-dimensional dynamical system for Rayleigh-Bénard convection subjected to magnetic field Journal of Applied Physics 113, 124902 (2013); 10.1063/1.4795264

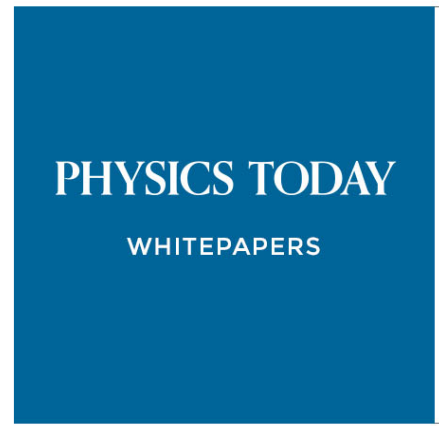

ADVANCED LIGHT CURE ADHESIVES

Take a closer look at what these environmentally friendly adhesive systems can do

\section{READ NOW}

PRESENTED BY Q8 MASTERBOND 


\title{
Asymptotic theory of wall-attached convection in a horizontal fluid layer with a vertical magnetic field
}

\author{
F. H. Busse ${ }^{a)}$ \\ Physikalisches Institut der Universität Bayreuth, D-95440 Bayreuth, Germany
}

(Received 20 July 2007; accepted 11 December 2007; published online 8 February 2008)

\begin{abstract}
A horizontal fluid layer heated from below in the presence of a vertical magnetic field is considered. A simple asymptotic analysis is presented which demonstrates that a convection mode attached to the side walls of the layer sets in at Rayleigh numbers much below those required for the onset of convection in the bulk of the layer. The analysis complements an earlier analysis by Houchens et al. [J. Fluid Mech. 469, 189 (2002)] which derived expressions for the critical Rayleigh number for the onset of convection in a vertical cylinder with an axial magnetic field in the cases of two aspect ratios. (C) 2008 American Institute of Physics. [DOI: 10.1063/1.2837175]
\end{abstract}

\section{INTRODUCTION}

Side walls of a fluid layer heated from below usually exert a stabilizing influence on the onset of convection. An exception to this rule has been found in the case of a rotating cylindrical layer where a side wall mode in the form of a traveling convection wave may set in at Rayleigh numbers $R$ below those required for the onset of convection in the bulk of the layer. This mode has been demonstrated experimentally by Zhong et al. ${ }^{1}$ and investigated theoretically by Goldstein $e t a l .{ }^{2}$ More recently another example for the destabilizing influence of the side wall of a cylindrical layer has been discovered by Houchens et al. ${ }^{3}$ (referred to by HWW in the following) in the case when a vertical magnetic field permeates the fluid.

The effect of a homogeneous vertical magnetic field on Rayleigh-Bénard convection in a horizontal fluid layer heated from below has been studied for a long time. Early results have been reviewed in Chandrasekhar's book ${ }^{4}$ (referred to by CH61 in the following). As emphasized in this book there exists a number of similarities between convection in the presence of a vertical magnetic field and convection in a layer rotating about a vertical axis. In this paper we would like to show that this similarity extends to the boundary layer nature of the side wall modes.

Using a boundary layer analysis Herrmann and Busse ${ }^{5}$ and Kuo and Cross ${ }^{6}$ have extended to the case of a straight side wall the numerical analysis of Goldstein et $a l^{2}$ of the onset of side wall convection in a cylindrical layer. Similarly, we intend to complement in this paper the numerical and semianalytical analyses of HWW with a boundary layer analysis at a straight side wall. This formulation of the problem permits the neglect of the aspect ratio parameter and simplifies the mathematical analysis.

In the following we first formulate the problem and then proceed through its analytical solution in Sec. III. The implications of the results will be discussed in the final section of the paper.

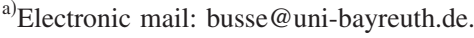

\section{MATHEMATICAL FORMULATION OF THE PROBLEM}

Since in the system considered here the fluid is electrically conducting, the electrical properties of the side wall enter the analysis in addition to the thermal ones. Following HWW we shall restrict the attention to the case when the electrical and the thermal conductivities are much lower than those of the fluid. The top and bottom surfaces are supposed to be stress-free and electrically insulating. In formulating the mathematical problem we consider a horizontal layer of height $d$ of an electrically conducting fluid as shown in Fig. 1. The constant temperatures, $T_{1}$ and $T_{2}$ with $T_{1}<T_{2}$, are prescribed at top and bottom boundaries, respectively. The layer is permeated by a homogeneous vertical magnetic field with the flux density $B_{0} . \kappa, \sigma$, and $\mu$ are thermal diffusivity, electrical conductivity, and magnetic permeability of the fluid. Using $d, d^{2} / \kappa, \Delta T \equiv T_{2}-T_{1}, B_{0}$ as scales for length, time, temperature, and magnetic field strength, respectively, the equation of motion, the continuity equation, the heat equation for the deviation $\Theta$ of the temperature from the static distribution, and the equation of magnetic induction can be written in the form

$$
\begin{aligned}
& \left(\nabla^{2}-P^{-1} \partial_{t}\right) \boldsymbol{u}+R \Theta \hat{\mathbf{k}}+Q\left(\hat{\mathbf{k}} \cdot \nabla+\frac{\kappa}{\lambda} \boldsymbol{b} \cdot \nabla\right) \boldsymbol{b} \\
& \quad=P^{-1}(\boldsymbol{u} \cdot \nabla \boldsymbol{u}+\nabla p), \\
& \nabla \cdot \boldsymbol{u}=0, \\
& \left(\partial_{t}+\boldsymbol{u} \cdot \nabla\right) \Theta=\boldsymbol{u} \cdot \hat{\mathbf{k}}+\nabla^{2} \Theta, \\
& \frac{\kappa}{\lambda}\left(\partial_{t} \boldsymbol{b}+\boldsymbol{u} \cdot \nabla \boldsymbol{b}-\boldsymbol{b} \cdot \nabla \boldsymbol{u}\right)=\nabla^{2} \boldsymbol{b}+\hat{\mathbf{k}} \cdot \nabla \boldsymbol{u}, \\
& \nabla \cdot \boldsymbol{b}=0,
\end{aligned}
$$

where $\hat{\mathbf{k}}$ is the unit vector opposite to the direction of gravity $g_{e}$. We have introduced the dimensionless magnetic field in the form $\boldsymbol{B}=\hat{\mathbf{k}}+\kappa \boldsymbol{b} / \lambda$, where $\lambda \equiv(\sigma \mu)^{-1}$ is the magnetic diffusivity. The Boussinesq approximation has been assumed. 


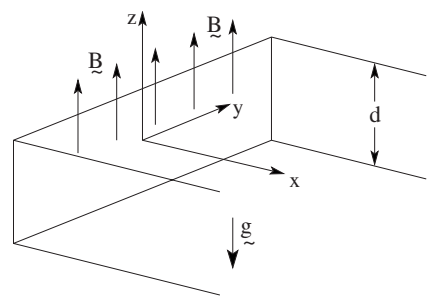

FIG. 1. Geometrical configuration of the problem of wall-attached convection in the presence of a vertical magnetic field.

The three dimensionless parameters, the Rayleigh, Prandtl, and Chandrasekhar numbers, are defined by

$$
R=\alpha g_{e} \Delta T d^{3} /(\nu \kappa), \quad P=\nu / \kappa, \quad Q=B_{0}^{2} d^{2} / \rho \mu \nu \lambda .
$$

In these expressions $\alpha$ is the coefficient of thermal expansion, $\nu$ is the kinematic viscosity, and $\rho$ is the density of the fluid.

It is convenient to use the following general representation for the solenoidal fields $\boldsymbol{u}$ and $\boldsymbol{b}$ :

$$
\begin{aligned}
& \boldsymbol{u}=\nabla \times(\nabla v \times \hat{\mathbf{i}})+\nabla w \times \hat{\mathbf{i}} \equiv \boldsymbol{\delta} v+\boldsymbol{\eta} w, \\
& \boldsymbol{b}=\nabla \times(\nabla h \times \hat{\mathbf{i}})+\nabla g \times \hat{\mathbf{i}} \equiv \boldsymbol{\delta} h+\boldsymbol{\eta} g,
\end{aligned}
$$

where $\hat{\mathbf{i}}$ is the unit vector normal to the side wall and directed into the fluid. In the following a Cartesian system of coordinates will be used with the $x$-coordinate in the direction of $\hat{\mathbf{i}}$ and the $z$-coordinate in the direction of $\hat{\mathbf{k}}$.

We anticipate that the onset of instability occurs in the form of monotonously growing modes and that the critical Rayleigh number will thus correspond to steady solutions of the linearized equations (1). We shall return to a discussion of the possibility of the onset of oscillatory convection at the end of this paper. Applying the operators $\boldsymbol{\delta}$ and $\boldsymbol{\eta}$ onto Eq. (1a), we obtain the following equations for the toroidal and poloidal components of the magnetic and velocity fields. In addition we rewrite Eq. (1c) for the temperature,

$$
\begin{aligned}
& \nabla^{4} \Delta_{2} v+R \partial_{x z}^{2} \Theta-Q \partial_{z z}^{2} \Delta_{2} v=0, \\
& \nabla^{2} \Delta_{2} w-R \partial_{y} \Theta+Q \partial_{z} \Delta_{2} g=0, \\
& \nabla^{2} \Theta-\partial_{y} w+\partial_{x z}^{2} v=0, \\
& \nabla^{2} \Delta_{2} g+\partial_{z} \Delta_{2} w=0
\end{aligned}
$$

where $\Delta_{2} \equiv \partial_{y y}^{2}+\partial_{z z}^{2}$. Eliminating $v, g$, and $\Theta$ from Eqs. (5) we obtain the following equation for $w$ :

$$
\left\{\left[\nabla^{4}-Q \partial_{z z}^{2}\right]^{2} \nabla^{2}-R\left[\nabla^{4}-Q \partial_{z z}^{2}\right]\left(\partial_{x x}^{2}+\partial_{y y}^{2}\right)\right\} w=0 .
$$

The boundary conditions at the stress-free and electrically insulating top and bottom boundaries and at the rigid sidewall are given by

$$
\begin{aligned}
& \partial_{z} v=\partial_{z z z}^{3} v=w=\partial_{z z}^{2} w=\Theta=\partial_{z} g=0 \quad \text { at } \quad z= \pm \frac{1}{2}, \\
& \partial_{x} v=v=w=\partial_{x} \Theta=g=0 \quad \text { at } \quad x=0 .
\end{aligned}
$$

It is worth noting that a consideration of the boundary condition for the poloidal function $h$ is not necessary since the equation $\nabla^{2} \Delta_{2} h+\partial_{z} \Delta_{2} v=0$ has been used directly to eliminate $h$ in the derivation of Eq. (5a).

\section{ANALYSIS AND RESULTS}

Without losing generality we propose a solution for Eq. (5) of the form

$$
\begin{aligned}
& v=\sum_{j=1}^{5} A_{j} \exp \left(-\mu_{j} x+i \beta y\right) \sin (\pi z), \\
& \Theta=\sum_{j=1}^{5} B_{j} \exp \left(-\mu_{j} x+i \beta y\right) \cos (\pi z), \\
& w=\sum_{j=1}^{5} C_{j} \exp \left(-\mu_{j} x+i \beta y\right) \cos (\pi z), \\
& g=\sum_{j=1}^{5} G_{j} \exp \left(-\mu_{j} x+i \beta y\right) \sin (\pi z) .
\end{aligned}
$$

The constants $\mu_{j}$ are the roots with positive real parts of the equation

$$
\hat{q}_{j} \equiv \mu_{j}^{2}-\beta^{2}-\pi^{2}
$$

where $\hat{q}_{j}, j=\{1, \ldots, 5\}$ are the five roots of

$$
\left[\hat{q}^{2}+Q \pi^{2}\right]^{2} \hat{q}-R\left[\hat{q}^{2}+Q \pi^{2}\right]\left(\hat{q}+\pi^{2}\right)=0 .
$$

The coefficients $A_{j}, B_{j}, C_{j}, G_{j}$, can be obtained in terms of five unknowns $D_{j}, j=\{1, \ldots, 5\}$,

$$
\begin{aligned}
& A_{j}=D_{j}\left\{\beta^{2} R \hat{q}_{j}-\left(\beta^{2}+\pi^{2}\right)\left[\hat{q}_{j}^{2}+Q \pi^{2}\right] \hat{q}_{j}\right\}, \\
& B_{j}=D_{j}\left(\beta^{2}+\pi^{2}\right)\left\{-\mu_{j} \pi\left[\hat{q}_{j}^{2}+Q \pi^{2}\right]\right\}, \\
& C_{j}=D_{j} \hat{q}_{j}\left[i \beta \mu_{j} \pi R\right], \\
& G_{j}=D_{j}\left[i \beta \mu_{j} \pi^{2} R\right] .
\end{aligned}
$$

The five unknowns $D_{j}$ are determined by the boundary conditions (8) at $x=0$,

$$
\begin{aligned}
& \sum_{j=1}^{5} \hat{q}_{j}\left\{\beta^{2} R-\left(\beta^{2}+\pi^{2}\right)\left[\hat{q}_{j}^{2}+Q \pi^{2}\right]\right\} D_{j}=0, \\
& \sum_{j=1}^{5} \mu_{j} \hat{q}_{j}\left\{\beta^{2} R-\left(\beta^{2}+\pi^{2}\right)\left[\hat{q}_{j}^{2}+Q \pi^{2}\right]\right\} D_{j}=0, \\
& \sum_{j=1}^{5} \mu_{j}\left\{-\mu_{j} \pi\left[\hat{q}_{j}^{2}+Q \pi^{2}\right]\right\} D_{j}=0, \\
& \sum_{j=1}^{5} \hat{q}_{j}\left[i \beta \mu_{j} \pi R\right] D_{j}=0, \\
& \sum_{j=1}^{5}\left[i \beta \mu_{j} \pi^{2} R\right] D_{j}=0 .
\end{aligned}
$$


In the limit of large $Q$ it is convenient to introduce rescaled quantities,

$$
\begin{aligned}
& r \equiv \varepsilon^{4} R, \quad \nu_{j} \equiv \varepsilon \mu_{j}, \\
& q_{j} \equiv \nu_{j}^{2}-\varepsilon^{2}\left(\beta^{2}+\pi^{2}\right), \quad j=1, \ldots, 5,
\end{aligned}
$$

where $\varepsilon$ is defined by $\varepsilon \equiv\left(Q \pi^{2}\right)^{-1 / 4}$. Using the rescaled variables we can rewrite Eq. (11) as

$$
\left(q^{2}+1\right)\left[\left(q^{2}+1\right) q-r\left(q+\varepsilon^{2} \pi^{2}\right)\right]=0 .
$$

The condition that the determinant of the homogeneous system (13) vanishes becomes

$$
D=\left|\begin{array}{ll}
\nu_{1}\left\{-\nu_{1} \pi\left[q_{1}^{2}+1\right]\right\} & \cdots \\
q_{1}\left\{\beta^{2} r-\left(\beta^{2}+\pi^{2}\right)\left[q_{1}^{2}+1\right]\right\} & \cdots \\
\nu_{1} q_{1}\left\{\beta^{2} r-\left(\beta^{2}+\pi^{2}\right)\left[q_{1}^{2}+1\right]\right\} & \cdots \\
q_{1}\left[i \beta \nu_{1} \pi r\right] & \cdots \\
i \beta \nu_{1} \pi^{2} r & \cdots
\end{array}\right|=0
$$

where the dots indicate the same columns as the first one except that the subscript 1 is replaced by $2,3,4$, and 5 , respectively.

In evaluating the roots of Eq. (15) we neglect terms of the order $\varepsilon^{2}$ smaller than those retained. The roots of Eq. (15) are thus given by

$$
q_{1}=\frac{\epsilon^{2} \pi^{2} r}{1-r}, \quad q_{2,3}= \pm i(1-r), \quad q_{4,5}= \pm i
$$

and the determinant (16) thus becomes

$$
\frac{D}{\beta^{2} r^{4} \pi^{4}} \equiv-\left|\begin{array}{ccccc}
-\nu_{1}^{2} & -\nu_{2}^{2} & -\nu_{3}^{2} & 0 & 0 \\
q_{1} B & -\pi^{2} q_{2} & -\pi^{2} q_{3} & q_{4} \beta^{2} & q_{5} \beta^{2} \\
\nu_{1} q_{1} B & -\pi^{2} \nu_{2} q_{2} & -\pi^{2} \nu_{3} q_{3} & \nu_{4} q_{4} \beta^{2} & \nu_{5} q_{5} \beta^{2} \\
q_{1} \nu_{1} r & \nu_{2} q_{2} & \nu_{3} q_{3} & \nu_{4} q_{4} & \nu_{5} q_{5} \\
\nu_{1} r & \nu_{2} & \nu_{3} & \nu_{4} & \nu_{5}
\end{array}\right|
$$

where the abbreviation $B \equiv\left[\beta^{2}(r-1)-\pi^{2}\right]$ has been introduced.

Using the relationships

$$
\nu_{1}=\varepsilon \sqrt{\beta^{2}+\pi^{2} /(1-r)}, \quad \nu_{j}^{2}=q_{j} \quad j=2, \ldots, 5,
$$

where again terms of the order $\varepsilon^{2}$ smaller than those retained have been neglected we get the following simplified expression for the determinant $D^{*} \equiv D /\left(\beta^{2} r^{4} \pi^{4} \nu_{1} q_{2} q_{3} q_{4} q_{5}\right)$ :

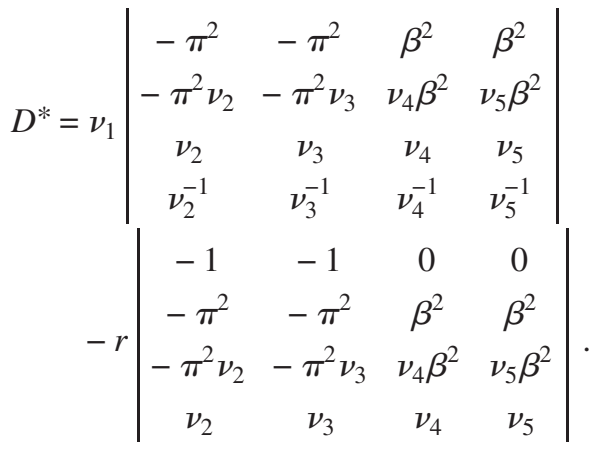

Here we have anticipated that $r$ will be of the order $\varepsilon$ and have included only the terms of lowest order in $\varepsilon$ in the first column of the determinant (18). The evaluation of expression (20) finally yields

$$
\begin{aligned}
D^{*}= & \left(\nu_{5}-\nu_{4}\right)\left(\nu_{3}-\nu_{2}\right)\left(\beta^{2}+\pi^{2}\right) \cdot\left\{\nu _ { 1 } \pi \left[\left(\beta^{2}+\pi^{2}\right)\right.\right. \\
& \left.\left.-\beta^{2} r / 4\right] \sqrt{2}-r \beta^{2}\right\} .
\end{aligned}
$$

After minimizing $r$ with respect to $\beta$ we obtain from the condition that $D^{*}$ vanishes the final result

$$
R_{c}=Q^{3 / 4} 3 \pi^{2} \sqrt{3 \pi / 2}\left(1+3 Q^{-1 / 4} \sqrt{3 \pi / 2}+\cdots\right),
$$

corresponding to

$$
\beta_{c}=\pi \sqrt{2},
$$

where for the derivation of the second term inside the brackets of expression (22) additional terms of the determinant (18) have been taken into account which had not been included in Eq. (20).

Setting $D_{1}=1$ we obtain the solution of Eqs. (13) in the form

$$
D_{2,3}=( \pm i-1) \frac{\nu_{1}^{2}}{2 r(1-r)}, \quad D_{4,5}( \pm i-1) \frac{\nu_{1}^{2} \pi^{2}}{2 r \beta^{2}} .
$$

For the temperature $\Theta$ we can derive a particularly simple expression,

$$
\begin{aligned}
\Theta= & -\exp (i \pi \sqrt{2} y) \cos (\pi z)\left(3 \pi^{2}\right)^{3 / 2} \\
& \cdot\left\{\exp \{-x \pi \sqrt{3}\}-\varepsilon \pi \sqrt{6} \exp \left\{-\frac{x}{\varepsilon \sqrt{2}}\right\} \cos \frac{x}{\varepsilon \sqrt{2}}\right\},
\end{aligned}
$$

the wavy bracket of which has been plotted in Fig. 2 .

\section{DISCUSSION}

The result (22) indicates an onset of convection at the side wall at a much lower Rayleigh number than is needed for the onset in the bulk of the convection layer. For large 


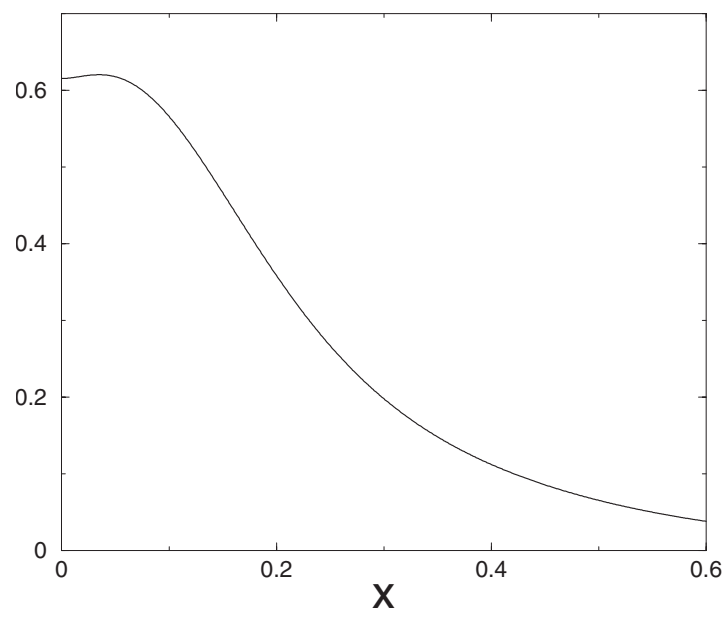

FIG. 2. Dependence of $\Theta$ as a function of $x$ at $z=y=0$ for $Q \pi^{2}=1600$.

values of $Q$ and for the same stress-free conditions (7) the Rayleigh number for the latter onset is given by

$$
R_{c}=Q \pi^{2}
$$

according to CH61. The result (22) agrees quite well with the finding $R_{c}=68.25 Q^{3 / 4}$ of HWW in the case of a cylinder with a radius equal to its height. The fact that this value exceeds the result (22) by about $6 \%$ must be expected since the aspect ratio is too small to realize the boundary layer character of the solution.

The lowering of the power of $Q$ with which the critical Rayleigh number in the presence of a side wall increases asymptotically is mainly caused by the fact that the temperature boundary condition permits convection to maintain a wavenumber of the order unity as shown by relationship (23). This property contrasts with the critical wavenumber $\alpha_{c}=\left(\pi^{4} Q / 2\right)^{1 / 6}$ corresponding to the result (26) for an unbounded convection layer.

A search of the literature has not revealed any observational evidence for the surprisingly low critical Rayleigh number (22). This is not unexpected since convection flows in liquid metals are notoriously difficult to observe. Most recent experiments ${ }^{7-9}$ have focused on measurements of the convective heat transport which also do not seem to have indicated evidence for onset of convection below the critical bulk Rayleigh number given by Eq. (26) for large $Q$. This effect may be caused by the relatively low contribution of the side wall mode to the heat transport or by the fact that the electrical and thermal conductivities of the stainless steel side walls were not sufficiently small in comparison to those of the metallic liquid.

Neither the Prandtl number $P$ nor the magnetic Prandtl number $P_{m}=\nu / \lambda$ have entered the analysis of this paper since only steady convection in the limit of vanishing amplitude has been considered. As discussed in CH61 oscillatory onset of convection becomes possible in the bulk of the convection layer for $P<P_{m}$. While the critical value of the Rayleigh number can be lower for oscillatory onset than for the monotonic one, it still grows in proportion to $Q$ for large $Q$. It is thus likely that even for $P<P_{m}$ the side wall mode will precede the onset of convection in the bulk. The possibility of the existence of an oscillatory side wall has not yet been investigated except in the limit $\kappa / \lambda \ll 1$ in which case a more general analysis of the kind described in this paper has eliminated such a possibility.

In contrast to a rotating convection layer where side wall modes propagate in the retrograde direction ${ }^{1,2,5,6}$ relative to the sense of rotation, side wall convection in the presence of a vertical magnetic field is steady. This property allows a direct application of the analysis to rectangular convection layers. Singularities at the corners of the rectangle will have a negligible effect as long as the horizontal dimensions are sufficiently large in comparison to the height of the layer.

The support of thermal convection by side walls in the presence of an applied magnetic field is of interest in the field of crystal growth. Magnetic fields have been used to suppress buoyancy driven flows in the case of Czochralski as well as Bridgman processes as discussed in the review. ${ }^{10}$ Numerical computations will be required for the investigation of these more general configurations. But the simple analytical model presented in this paper will be useful for the elucidation of the mathematical structure of the problem.

\section{ACKNOWLEDGMENTS}

The support by NASA through Grant No. NNG04GG07G of the research reported in this letter is gratefully acknowledged.

${ }^{1}$ F. Zhong, R. E. Ecke, and V. Steinberg, "Asymmetric modes and the transition to vortex structures in rotating Rayleigh-Bénard convection," Phys. Rev. Lett. 67, 2473 (1991).

${ }^{2}$ H. F. Goldstein, E. Knobloch, I. Mercader, and M. Net, "Convection in a rotating cylinder. Part I. Linear theory for moderate Prandtl numbers," J. Fluid Mech. 248, 583 (1993).

${ }^{3}$ B. C. Houchens, L. M. Witkowski, and J. S. Walker, "Rayleigh-Benard instability in a vertical cylinder with a vertical magnetic field," J. Fluid Mech. 469, 189 (2002).

${ }^{4}$ S. Chandrasekhar, Hydrodynamic and Hydromagnetic Stability (Clarendon, Oxford, 1961).

${ }^{5}$ J. Herrmann and F. H. Busse, "Asymptotic theory of wall attached convection in a rotating fluid layer," J. Fluid Mech. 255, 183 (1993).

${ }^{6}$ E. Y. Kuo and M. C. Cross, "Travelling-wave wall states in rotating Rayleigh-Benard convection," Phys. Rev. E 47, R2245 (1993).

${ }^{7}$ S. Cioni, S. Chaumat, and J. Sommeria, "Effect of a vertical magnetic field on turbulent Rayleigh-Bénard convection," Phys. Rev. E 62, R4520 (2000).

${ }^{8} \mathrm{U}$. Burr and U. Müller, "Rayleigh-Bénard convection in liquid metal layers under the influence of a vertical magnetic field," Phys. Fluids 13, 3247 (2001).

${ }^{9}$ J. M. Aurnou and P. L. Olson, "Experiments on Rayleigh-Bénard convection, magnetoconvection and rotating magnetoconvection in liquid gallium," J. Fluid Mech. 430, 283 (2001).

${ }^{10}$ R. W. Series and D. T. J. Hurle, "The use of magnetic fields in semiconductor crystal growth,” J. Cryst. Growth 113, 305 (1991). 